\title{
Editorial
}

\section{A Bim-activating mould}

\author{
G Häcker ${ }^{*}, 1$ \\ Cell Death and Differentiation (2013) 20, 1289-1290; doi:10.1038/cdd.2013.98
}

Aspergillus fumigatus is a mould found basically everywhere: in water, soil, food, in decaying matter (for instance in your rubbish bin) and floating through the air. ${ }^{1}$ The genus Aspergillus (and here by far the most common one is A. fumigatus) can also cause invasive aspergillosis, a difficult-to-diagnose and life-threatening condition in immunosuppressed patients ${ }^{2}$ (Figure 1). A. fumigatus has a well-stocked arsenal of factors that are thought to contribute to its virulence. ${ }^{3}$ In this issue of the journal, Geissler et al. ${ }^{4}$ show that one of the prominent toxins of $A$. fumigatus, gliotoxin, induces apoptosis in a number of mammalian cells through a signalling pathway involving the JNK-dependent phosphorylation of the $\mathrm{BH}$-only protein Bim. This finding provides new insight into the activation of Bim and also adds to our understanding of the details of our co-existence with a ubiquitous fungus.

Bim is perhaps the most prominent of the BH3-only proteins. Judging by the phenotype of the various genedeficient mouse strains generated, Bim is the most important $\mathrm{BH} 3-$ only protein in the immune system, where it participates in apoptosis induction in most cell types and most situations tested. ${ }^{5,6} \mathrm{BH} 3$-only proteins are considered the sensors of the mitochondrial apoptosis pathway: ${ }^{7}$ they are the most upstream components in the Bcl-2 family, which regulates mitochondrial apoptosis.

The Bcl-2-protein family has three subgroups. The effectors, Bax and Bak, release cytochrome- $c$ from the mitochondria. The BH3-only proteins can, when experimentally overexpressed, with varying potency activate Bax and Bak; in a world of numerous molecular uncertainties like mitochondrial apoptosis, the knowledge is reassuringly stable that BH3-only proteins require Bax or Bak to induce apoptosis. The third group of Bcl-2 family proteins, the anti-apoptotic proteins (including $\mathrm{Bcl}-2$ itself), block apoptosis by binding to the pro-apoptotic ones. They can bind the members of both groups, but the neutralisation of $\mathrm{BH} 3-$ only proteins seems to be more important than the binding of Bax and Bak. ${ }^{8}$

Besides this sensing of apoptotic stimuli by $\mathrm{BH}$-only proteins, stimuli may also be sensed by anti-apoptotic proteins. This has been most extensively studied for Mcl-1, which is lost in response to many signals, and this loss may free $\mathrm{BH} 3-$ only proteins for the activation of Bax/Bak. ${ }^{9}$ On this framework, we have to identify the molecular mechanisms of how the chain of events in the Bcl-2 family is set off, starting from the generation of active $\mathrm{BH} 3-o n l y$ proteins, and here
Geissler et al. ${ }^{4}$ provide new information. Their data argue for a role of Bim in the sensing of signals delivered by the $\mathrm{c}$-jun $\mathrm{NH}_{2}$-terminal kinases 1 and 2 (JNK1/2).

JNKs are MAP kinases. They are activated by cytokines and by stress stimuli and can initiate a number of biological responses of the cell such as differentiation, inflammatory responses, proliferation and, relevant here, apoptosis. ${ }^{10}$ Many reports demonstrate that JNKs can activate mitochondrial apoptosis (reviewed in Weston and Davis ${ }^{10}$ ). However, the proposed mechanisms differ substantially and range from inactivation by phosphorylation of anti-apoptotic proteins over the transcriptional induction of Bim to its direct phosphorylation. A JNK-phosphorylation site (T112) has been identified previously in Bim, but a knock-in mouse where this site had been mutated had only very modest changes in apoptosis. $^{11}$

Geissler et al. ${ }^{4}$ now show that gliotoxin-induced apoptosis to a substantial degree depends on the presence of Bim. They also confirm their earlier results ${ }^{12}$ that gliotoxin requires Bak for full activity, whereas the contribution of Bax is only minor. Importantly, this recent work identifies two additional JNK-phosphorylation sites in Bim and demonstrates that all three sites have to be mutated to prevent gliotoxin-induced apoptosis. The approach taken may have technical issues because for this analysis mutant Bim forms were reintroduced into Bim-deficient cells under a retroviral promoter, which may subject the cells to a selection bias. However, taking the data at face value, they show impressively that triple-mutated Bim is inactive at transmitting the gliotoxinJNK-initiated apoptotic stimulus to Bak.

What is happening at the molecular level? The data indicate that gliotoxin induces JNK activation from the plasma membrane (at variance with an earlier suggestion that it directly activates Bak at mitochondria ${ }^{12}$ ) and that this activation is necessary (although it may not be sufficient) to initiate Bak activation. It is suggested that the phosphorylated $\mathrm{Bim}$ is released from the cytoskeleton more efficiently. Such release was suggested 14 years ago ${ }^{13}$ but has not received much experimental support since, and more recent data dispute at least the generality of this mechanism. ${ }^{14}$ Geissler et al. ${ }^{4}$ also show that gliotoxin treatment fails to increase $\mathrm{Bcl}-2$ or $\mathrm{Bcl}-\mathrm{X}_{\mathrm{L}}$ precipitation with Bim-phosphorylation site mutants, whereas it does for wt Bim. One way of interpreting this piece of data is that mutant Bim is less active as it cannot inhibit these anti-apoptotic proteins as strongly.

\footnotetext{
${ }^{1}$ Department of Medical Microbiology and Hygiene, University Medical Center Freiburg, Freiburg, Germany

*Corresponding author: G Häcker, Department of Medical Microbiology and Hygiene, University Medical Center Freiburg, Hermann-Herder-Str. 11, D-79104 Freiburg, Germany. Tel: +49 761203 6531; Fax: +49 761203 6551; E-mail: georg.haecker@ uniklinik-freiburg.de
} 

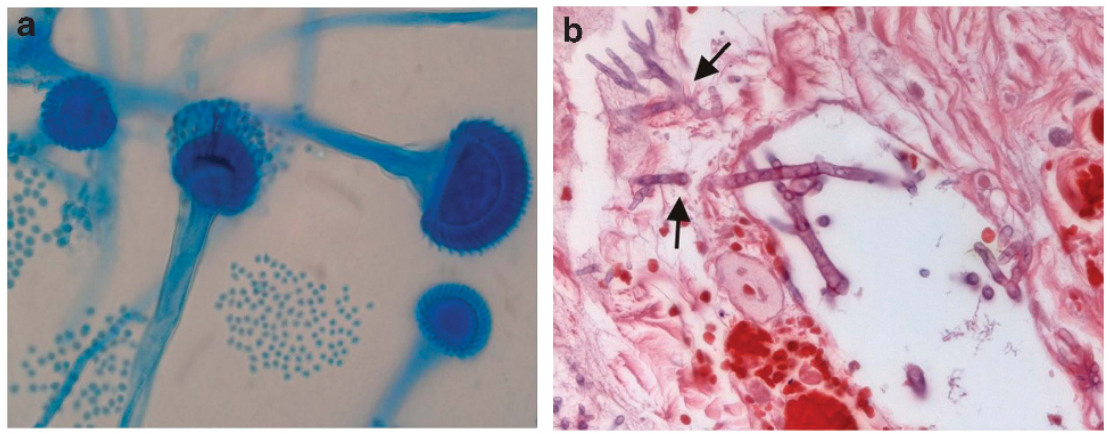

Figure 1 (a) Microscopy from a culture of $A$. fumigatus (lactophenol blue stain). The name Aspergillus was introduced in 1729 because of the similarity to the aspergillum, the implement used to sprinkle the holy water. (b) Invasive aspergillosis. Aspergillus hyphae invade in this case the gut epithelium (arrows). Credits: Annerose Serr (A) and Annette Schmitt-Gräff (B), University Medical Center Freiburg

However, it is very much under dispute whether Bim-Bcl-2binding means that Bim inhibits Bcl-2 or that Bcl-2 inhibits Bim. ${ }^{15}$ The binding studies by Geissler et al. ${ }^{4}$ may therefore reflect any 'activation' of Bim and the associated binding of Bim to $\mathrm{Bcl}-2$, and the observed differences may reflect reduced upstream signals. Whether this is linked to release of Bim from the cytoskeleton, from anti-apoptotic proteins (which may be downregulated as a response to gliotoxin) or any other molecular event is not clear at this stage. That the molecular details of JNK-Bim-dependent, gliotoxin-triggered apoptosis are uncertain is also highlighted by its reliance on Bak. Bim can efficiently also activate Bax. ${ }^{7}$ That it does not in this study indicates that other mechanisms are relevant that have not yet been discovered. However, the data provide one of the few examples where upstream signalling is with only few gaps linked to the $\mathrm{Bcl}-2$ family.

Gliotoxin from Aspergillus thus induces Bim/Bak-dependent apoptosis. Although the evidence that gliotoxin is an important virulence factor is equivocal, ${ }^{16}$ such apoptosis induction may well be relevant to human invasive aspergillosis. It is unlikely that apoptosis induction, or any other effect on human cells, is the benefit that Aspergillus derives from gliotoxin production. It is certainly correct that $A$. fumigatus is the most common mould in invasive human disease, that the incidence of invasive aspergillosis has risen substantially in recent years and that these cases have a poor prognosis. However, given the ubiquitous existence of Aspergillus in nature and the rarity of aspergillosis in immunocompetent hosts, it seems very unlikely that the capacity for human infection is a relevant factor driving Aspergillus evolution. Gliotoxin may therefore have functions outside the human host.
A major risk factor predisposing humans to aspergillosis is the lack of functional neutrophils, and in neutropenic mice the lack of gliotoxin did not alter Aspergillus virulence (although it did in non-neutropenic mice). ${ }^{3,16}$ This does not exclude a relevant role of gliotoxin in human invasive infections. Gliotoxin-dependent apoptosis induction may well affect severity and course of Aspergillus infection, for instance by destroying lung epithelium or by killing alveolar macrophages. Just as Aspergillus does not rely on humans for its niche in nature but can nevertheless cause severe infections, gliotoxin may have another function but may, given the chance, have effects on the human host. In any case, the report by Geissler et al. ${ }^{4}$ uncovers new ways in the multi-facetted interaction of human cells with their environment and may have hit upon a new way of stress response that is relevant to a broader array of situations.

1. Summerbell R, Howard DH (eds). Pathogenic Fungi in Humans and Animals. 2nd edn. Marcel Dekker: New York, 2003. pp 237-498.

2. Walsh TJ et al. Clin Infect Dis 2008; 46: 327-360.

3. Abad A et al. Revista iberoamericana de micologia 2010; 27: 155-182.

4. Geissler A et al. Cell Death Differ 2013; 20: 1317-1329.

5. Strasser A. Nat Rev Immunol 2005; 5: 189-200.

6. Happo L, Strasser A, Cory S. J Cell Sci 2012; 125: 1081-1087.

7. Chipuk JE et al. Mol Cell 2010; 37: 299-310.

8. Llambi F et al. Mol Cell 2011; 44: 517-531.

9. Stewart DP et al. Mol Cell Biol 2010; 30: 3099-3110.

10. Weston CR, Davis RJ. Curr Opin Cell Biol 2007; 19: 142-149.

11. Hubner A et al. Mol Cell 2008; 30: 415-425.

12. Pardo J et al. J Cell Biol 2006; 174: 509-519.

13. Puthalakath $\mathrm{H}$ et al. MolCell 1999; 3: 287-296.

14. Wilfling $\mathrm{F}$ et al. Cell Death Differ 2012; 19: 1328-1336.

15. Villunger $A$ et al. Cell Death Differ 2011; 18: 1545-1546

16. Scharf DH et al. Appl Microbiol Biotechnol 2012; 93: 467-472. 